\title{
Oxidised, glycated LDL selectively influences tissue inhibitor of metalloproteinase-3 gene expression and protein production in human retinal capillary pericytes
}

\author{
J. L. Barth • Y. Yu • W. Song $\cdot$ K. Lu • A. Dashti • \\ Y. Huang • W. S. Argraves $\cdot$ T. J. Lyons
}

Received: 14 February 2007 / Accepted: 22 June 2007 / Published online: 4 August 2007

(C) Springer-Verlag 2007

\begin{abstract}
Aims/hypothesis Matrix metalloproteinases (MMPs) and their natural inhibitors, tissue inhibitor of metalloproteinases (TIMPs), regulate important biological processes including the homeostasis of the extracellular matrix, proteolysis of cell surface proteins, proteinase zymogen activation, angiogenesis and inflammation. Studies have shown that their balance is altered in retinal microvascular tissues in diabetes. Since LDLs modified by oxidation/ glycation are implicated in the pathogenesis of diabetic vascular complications, we examined the effects of modified LDL on the gene expression and protein production of MMPs and TIMPs in retinal pericytes.
\end{abstract}

J. L. Barth, Y. Yu and W. Song contributed equally to this paper.

J. L. Barth · W. S. Argraves

Department of Cell Biology and Anatomy,

Medical University of South Carolina,

Charleston, SC, USA

Y. Yu $\cdot$ W. Song $\cdot$ K. Lu $\cdot$ A. Dashti $\cdot$ T. J. Lyons $(\bowtie)$

Section of Endocrinology and Diabetes,

University of Oklahoma Health Sciences Center, WP1345,

Oklahoma City, OK 73104, USA

e-mail: timothy-lyons@ouhsc.edu

\section{Y. Huang}

Ralph H. Johnson VA Medical Center,

Charleston, SC, USA

\section{Y. Huang}

Division of Endocrinology, Diabetes and Medical Genetics,

Medical University of South Carolina,

Charleston, SC, USA

T. J. Lyons

VA Medical Center,

Oklahoma City, OK, USA
Methods Quiescent human retinal pericytes were exposed to native LDL (N-LDL), glycated LDL (G-LDL) and heavily oxidised and glycated LDL (HOG-LDL) for $24 \mathrm{~h}$. We studied the expression of the genes encoding MMPs and TIMPs mRNAs by analysis of microarray data and quantitative PCR, and protein levels by immunoblotting and ELISA.

Results Microarray analysis showed that $M M P 1, M M P 2$, MMP11, MMP14 and MMP25 and TIMP1, TIMP2, TIMP3 and TIMP4 were expressed in pericytes. Of these, only TIMP3 mRNA showed altered regulation, being expressed at significantly lower levels in response to HOG-vs N-LDL. Quantitative PCR and immunoblotting of cell/matrix proteins confirmed the reduction in TIMP3 mRNA and protein in response to HOG-LDL. In contrast to cellular TIMP3 protein, analysis of secreted TIMP1, TIMP2, MMP1 and collagenase activity indicated no changes in their production in response to modified LDL. Combined treatment with $\mathrm{N}-$ and HOG-LDL restored TIMP3 mRNA expression to a level comparable with that after N-LDL alone.

Conclusions/interpretation Among the genes encoding for MMPs and TIMPs expressed in retinal pericytes, TIMP3 is uniquely regulated by HOG-LDL. Reduced TIMP3 expression might contribute to microvascular abnormalities in diabetic retinopathy.

Keywords Diabetic retinopathy - Gene array - Glycation . Lipoprotein $\cdot$ Metalloproteinase $\cdot$ Oxidation

$\begin{array}{ll}\text { Abbreviations } \\ \text { ApoB } & \text { apolipoprotein B } \\ \text { ECM } & \text { extracellular matrix } \\ \text { G-LDL } & \text { glycated low-density lipoprotein } \\ \text { HOG-LDL } & \begin{array}{l}\text { heavily oxidised and glycated low-density } \\ \text { lipoprotein }\end{array}\end{array}$




$\begin{array}{ll}\text { MMP } & \text { matrix metalloproteinase } \\ \text { N-LDL } & \text { native low-density lipoprotein } \\ \text { QPCR } & \text { quantitative PCR } \\ \text { RSM } & \text { reduced-serum medium } \\ \text { SFM } & \text { serum-free medium } \\ \text { TIMP } & \text { tissue inhibitor of metalloproteinase } \\ \text { VEGF } & \text { vascular endothelial growth factor }\end{array}$

\section{Introduction}

Matrix metalloproteinases (MMPs) and their natural inhibitors, tissue inhibitors of metalloproteinases (TIMPs), regulate important biological functions, including the integrity of the extracellular matrix (ECM), the proteolysis of cell surface proteins, zymogen activation, angiogenesis and inflammation [1]. There is evidence that altered tissue levels of MMPs or TIMPs are involved in retinal diseases. For instance, retinal pigment epithelium cells from patients with age-related macular degeneration secrete two- to threefold more MMP2 than those from healthy donors [2]. Similarly, MMP2 is nearly doubled in retinal pigment epithelium-associated interphotoreceptor matrix from the eyes of patients with age-related macular degeneration compared with normal controls [3]. TIMP3 gene mutations have been implicated in Sorsby fundus dystrophy [4], although the exact mechanism is unknown.

Alterations of MMP-TIMP balance also occur in diabetic retinopathy, a disease characterised by microvascular abnormalities (i.e. early-stage pericyte dropout and vascular leakage, and late-stage neovascularisation) $[5,6]$. Grant et al. [5] reported constitutive mRNA expression of MMP2, MMP9, TIMP1 and TIMP2 in both non-diabetic and diabetic human retinal endothelial cells, which were not affected by a high glucose concentration; however, MMP2 protein activity was modified by different concentrations of glucose. Das et al. [6] found significantly elevated levels of MMP2 and MMP9 in the epiretinal neovascular membranes from patients with proliferative diabetic retinopathy in comparison with levels in normal retinae. Elevated levels or activities of MMPs have also been associated with loss of integrity of the blood-retina barrier [7] and vitreous haemorrhage [8] in diabetes. Despite these findings, the underlying pathogenic factors and cellular mechanisms are obscure.

Modified LDL, e.g. LDL modified by oxidation and glycation, is elevated in diabetic plasma [9-11] and is established as playing an important role in atherogenesis in both diabetic and non-diabetic patients [12]. However, its role in diabetic retinopathy is less clear. We have hypothesised that glycated and oxidised LDL is involved in the pathogenesis of diabetic retinopathy [13]. In support of this, Cusick et al. [14] have demonstrated dense concentrations of apolipoprotein B (ApoB) with co-localisation of macrophages around retinal vessels in human diabetic retinae, and we have demonstrated that modified LDLs cause cytotoxic effects in retinal capillary pericytes and endothelial cells $[13,15]$.

In a previous gene array study, we found that TIMP3 was dysregulated in human retinal pericytes upon exposure to modified LDL [16]. Here we report an analysis of our existing DNA microarray data to investigate comprehensively the effects of modified human LDL on all genes encoding MMPs and TIMPs expressed in human retinal pericytes, with validation by real-time quantitative PCR (QPCR) and immunoblotting experiments. Three forms of LDL were employed: native LDL (N-LDL), glycated LDL (G-LDL) and heavily oxidised and glycated LDL (HOGLDL). These simulated, respectively, normal LDL found in human plasma, mildly modified LDL (i.e. glycated but not oxidised LDL) present in the plasma of diabetic patients, and severely modified LDL, which is mainly formed after prolonged extravasation.

\section{Methods}

Analysis of DNA microarray data Our DNA microarray analysis of human retinal pericytes treated with modified LDL has been reported previously [16]. Briefly, in four separate experiments, pericytes treated for $24 \mathrm{~h}$ with $\mathrm{N}-$, $\mathrm{G}-$ or HOG-LDL were analysed by hybridisation to human U95Av2 GeneChips (Affymetrix, Santa Clara, CA, USA). Microarray files of this project can be accessed using the target identifier_1046387919.146377 from the Medical University of South Carolina (MUSC) DNA microarray database (http://www.proteogenomics.musc.edu/pss/home. php). Sixty genes responded differently to HOG- vs NLDL. Of these, the only representative of the MMP/TIMP family was TIMP3, whose expression was significantly downregulated by HOG- vs N-LDL.

For the present study, raw hybridisation data (.CEL files) associated with our microarray study [16] were analysed using dChip software [17]. Each experiment was treated as independent so that there were four replicates of each treatment. Hybridisation intensities were normalised using the invariant gene set expression model. Model-based expression indices were calculated based on the perfect match-only model. The Affymetrix tool NetAffx was used to find all genes encoding TIMPs and MMPs represented on this GeneChip. Those genes not confidently scored 'present' (Affymetrix MAS5.0 detection, $p<0.04$ ) for at least two of four replicate tests for any experimental condition were deemed unexpressed or undetectable and were excluded. 
LDL preparation, modification and characterisation For preparation of native and modified LDLs, LDL was isolated from fasting, healthy, normolipaemic, non-diabetic participants aged 20-40 years taking neither prescribed medications nor antioxidant vitamin supplements. The study was approved by the Institutional Review Boards of MUSC and the University of Oklahoma Health Sciences Center (OUHSC) and conformed to the tenets of the Declaration of Helsinki. Informed consent was obtained from all volunteers. N-LDL $(d=1.019-1.063 \mathrm{~g} / \mathrm{ml})$ was prepared by sequential ultracentrifugation, pooled, and modified in vitro as described [18]. G-LDL was prepared by incubating $\mathrm{N}-\mathrm{LDL}$ in freshly prepared $50 \mathrm{mmol} / 1$ glucose $\left(72 \mathrm{~h}, 37^{\circ} \mathrm{C}\right)$ under antioxidant conditions $(1 \mathrm{mmol} / \mathrm{l}$ diethylene triamine pentaacetic acid [DTPA], under nitrogen). HOG-LDL was prepared from G-LDL by incubation in $10 \mu \mathrm{mol} / 1 \mathrm{CuCl}_{2}$ $\left(24 \mathrm{~h}, 37^{\circ} \mathrm{C}\right)$ under air. The protein content of LDL was determined using a bicinchoninic acid assay kit (Pierce, Rockford, IL, USA). Native and modified LDLs were characterised by agarose gel electrophoresis (LIPOEPG; Beckman, Fullerton, CA, USA), fluorescence at $360 \mathrm{~nm}$ excitation and $430 \mathrm{~nm}$ emission (Gilford Fluorimeter IV, Oberlin, OH, USA) and absorbance at $234 \mathrm{~nm}$ in a Beckman DU650 spectrophotometer, confirming that the products were of a quality comparable with that in previous reports [18].

Cell culture and treatment Human retinal pericytes (Clonetics, Walkersville, MD, USA) were maintained and treated as described previously [16]. Briefly, cells were grown at $37^{\circ} \mathrm{C}$ with $5 \% \mathrm{CO}_{2}$ in medium containing $5 \%$ fetal bovine serum, $0.04 \%$ hydrocortisone, $0.4 \%$ human fibroblast growth factor B (hFGF-B), $0.1 \%$ vascular endothelial growth factor (VEGF), $0.1 \% R^{3}$-IGF-1, $0.1 \%$ ascorbic acid, $0.1 \%$ human epidermal growth factor (hEGF) and $0.1 \%$ aqueous solution of gentamicin sulphate and amphotericin-B (GA1000). At $85 \%$ confluence, cells were exposed to serum-free medium (SFM) for $24 \mathrm{~h}$ to induce quiescence, then treated for a further $24 \mathrm{~h}$ with $\mathrm{N}-, \mathrm{G}-$ or HOG-LDL (spiked into medium). For experiments involving combined treatment with $\mathrm{N}-$ and HOG-LDL, the two LDL treatments were simultaneously administered at $100 \mu \mathrm{g}$ protein $/ \mathrm{ml}$ each.

Real-time quantitative PCR Primer sequences (forward, 5'GCG TCT ATG ATG GCA AGA TGT-3'; reverse, 5'-GTC ACA AAG CAA GGC AGG TAG-3') for the QPCR analysis of TIMP3 were designed using Primer3 (http:// www.frodo.wi.mit.edu/cgi-bin/primer3/primer3.cgi). Aliquots of cDNA samples for the microarray study [16] were used to confirm the result. For analysis of TIMP3 in response to differing concentrations of N-and HOG-LDL and to co-incubation with $\mathrm{N}-$ and HOG-LDL, total RNA was isolated from treated cells and first-strand cDNA was prepared. cDNA templates were diluted 1:160 and then used in amplification reactions performed in the Smart Cycler PCR amplifier (Cepheid, Sunnyvale, CA, USA). Amplification conditions were $95^{\circ} \mathrm{C}$ for 10 min followed by 40 cycles at $95^{\circ} \mathrm{C}$ for $15 \mathrm{~s}$ and $60^{\circ} \mathrm{C}$ for $60 \mathrm{~s}$. SYBR Green PCR MasterMix kit (Applied Biosystems, Foster City, CA, USA) was used for fluorescence labelling. Melting curve analysis was performed following amplification. Amplicon size and reaction specificity were confirmed by electrophoretic separation on $2.5 \%$ agarose gels. Each experiment was performed three times. Glyceraldehyde-3-phosphate dehydrogenase was included for standardisation of expression measurements.

Immunoblotting For immunoblot detection of TIMP3 protein, cells treated with $\mathrm{N}-$, G- or HOG-LDL were washed three times with PBS, lysed on ice with $100 \mu 1$ lysis buffer $(62.5 \mathrm{mmol} / \mathrm{l}$ Tris- $\mathrm{HCl}, \mathrm{pH} 6.8,10 \%$ glycerol and $1 \%$ Triton $\mathrm{X}-100$ ) and sonicated for $15 \mathrm{~s}$. Protein was quantified by the bicinchoninic acid assay. A sample $(2 \mu \mathrm{g})$ was subjected to electrophoresis using a 4-20\% Tris-glycine, Novex precast SDS-PAGE gel (Invitrogen, Carlsbad, CA, USA) under reducing conditions, and was transferred to a polyvinylidene fluoride membrane (Pall, Ann Arbor, MI, USA). The membrane was blocked with $3 \%$ non-fat Carnation milk in TBS and $0.05 \%$ Tween 20 , and then incubated overnight at $4^{\circ} \mathrm{C}$ with human TIMP3 monoclonal antibody (AB-1, clone 136-13H4; Calbiochem, San Diego, CA, USA) diluted 1:100 in Tris-buffered saline and low-fat $(0.5 \%)$ milk. The membrane was washed three times with TBS, incubated with antimouse secondary peroxidise-labelled IgG (IgG-POD) Fab fragment (Roche Diagnostics, Indianapolis, IN, USA) for $1 \mathrm{~h}$, and then subjected to chemiluminescent detection.

ELISA and collagenase activity assay ELISA kits (Oncogene, Boston, MA, USA) were used to measure the levels of TIMP1, TIMP2 and MMP1 in conditioned culture medium. It is established that TIMP3 is not released into culture medium, but only into the ECM [19], and our preliminary data confirmed that TIMP3 was undetectable in cell culture medium. Collagenase activity in conditioned medium was quantified using the EnzChekTM Gelatinase/Collagenase assay kit (Molecular Probes, Eugene, OR, USA).

Statistical analysis Statistical significance was assessed using the unpaired, two-tailed Student's $t$ test. For comparisons of microarray data involving TIMPs and MMPs, $p$ values were corrected for multiple testing using the Bonferroni adjustment. Significance was assigned at $p<0.05$.

\section{Results}

Reduced TIMP3 gene expression in retinal pericytes in response to HOG-LDL To investigate the cellular expres- 
sion of genes encoding for TIMPs and MMPs following exposure to modified LDL, analysis was performed on the DNA microarray data generated previously [16], in which human retinal pericytes treated with $\mathrm{N}-$, G- or HOG-LDL $(100 \mu \mathrm{g}$ protein $/ \mathrm{ml}, 24 \mathrm{~h})$ were analysed by hybridisation to Affymetrix U95Av2 GeneChips. TIMP1, TIMP2, TIMP3 and TIMP4 and 19 genes encoding MMPs were included among the $>10,000$ genes represented on this array. Results showed that all four TIMP genes and five of the MMP genes (MMP1, MMP2, MMP11, MMP14 and MMP25) were expressed in pericytes (Table 1). TIMP3 expression decreased by approximately $60 \%$ in response to HOG- vs N-LDL $(p<0.001, n=4)$, whereas expression of all other genes encoding TIMPs and MMPs remained largely unchanged. G-LDL did not change the expression of any of these genes significantly compared with N-LDL ( $p>$ $0.05, n=4)$. Thus, among the genes encoding TIMPs and MMPs expressed, TIMP3 is unique in its response to HOGLDL. To validate the distinct effects of HOG-LDL on TIMP3 expression, QPCR analysis was performed on the same cDNA samples from the microarray study. As shown in Fig. 1a, HOG-LDL inhibited TIMP3 mRNA expression by $68 \%$, consistent with the microarray data. In separate, duplicate experiments, we determined the concentration relations of this response (Fig. 1b). Cells were treated with 50-200 $\mu \mathrm{g}$ protein $/ \mathrm{ml}$ of $\mathrm{N}-$ or HOG-LDL for $24 \mathrm{~h}$. By QPCR, levels of TIMP3 expression were similar in response to the three concentrations of N-LDL, but were significantly reduced by HOG-LDL in a concentrationrelated fashion, i.e. there were reductions of 63,75 and
$78 \%$ for 50,100 and 200 protein/ml of HOG-LDL respectively, compared with N-LDL.

To determine whether decreased TIMP3 mRNA levels in response to HOG-LDL could be affected by addition of NLDL, both lipoproteins (each at $100 \mu \mathrm{g}$ protein $/ \mathrm{ml}, 24 \mathrm{~h}$ ) were applied in combination to pericyte cultures. By QPCR, TIMP3 expression in the presence of both $\mathrm{N}-$ and HOG-LDL was similar to that in the presence of N-LDL alone (Fig. 1c), indicating that the effect of HOG-LDL was completely reversed by addition of N-LDL. This suggests that modification of the LDL may inactivate it with respect to stimulating TIMP3 expression.

In the course of these experiments, we also employed lipoprotein-free control conditions. TIMP3 expression in SFM control conditions proved variable in comparison with expression in the presence of N-LDL, perhaps reflecting varying responses to nutrient deprivation. Under reduced serum conditions $(0.5 \%$ serum; reduced-serum medium [RSM]), TIMP3 expression was similar in the presence and absence of N-LDL (data not shown).

TIMP3 production is reduced in retinal pericytes in response to $H O G-L D L$ To determine the effects of modified LDL on TIMP3 production, immunoblotting of the wholecell lysate was performed in pericytes after treatment with N-, G- or HOG-LDL (100 $\mu \mathrm{g}$ protein/ml, 24 h). As shown in Fig. 2a, TIMP3 was readily detectable after N-LDL treatment but was significantly lower in response to HOGLDL. The extent of the reduction in TIMP3 level was comparable with that of the mRNA reduction (Table 1,

Table 1 mRNA expression of the genes encoding TIMPs and MMPs in cultured human retinal pericytes in response to treatment with native and modified LDL

\begin{tabular}{|c|c|c|c|c|c|c|c|}
\hline \multirow[t]{2}{*}{ Gene symbol } & \multirow[t]{2}{*}{ Alternative name } & \multirow[t]{2}{*}{ Probe set $\mathrm{ID}^{\mathrm{a}}$} & \multicolumn{3}{|c|}{ Hybridisation intensity ${ }^{\mathrm{b}}$} & \multicolumn{2}{|c|}{ Fold change } \\
\hline & & & $\mathrm{N}-\mathrm{LDL}^{\mathrm{c}}$ & G-LDL ${ }^{c}$ & HOG-LDL ${ }^{c}$ & $\begin{array}{l}\text { G-LDL vs } \\
\text { N-LDL }\end{array}$ & $\begin{array}{l}\text { HOG-LDL vs } \\
\text { N-LDL }\end{array}$ \\
\hline TIMPI & & 1693_s_at & $8156 \pm 511$ & $7958 \pm 470$ & $6910 \pm 618$ & -1.02 & -1.18 \\
\hline TIMP2 & & 1375_s_at & $406 \pm 47$ & $413 \pm 56$ & $416 \pm 23$ & 1.02 & 1.03 \\
\hline TIMP2 & & 34722_at & $206 \pm 31$ & $200 \pm 32$ & $202 \pm 20$ & -1.03 & -1.02 \\
\hline TIMP3 & & 1034_at & $1011 \pm 163$ & $843 \pm 123$ & $398 \pm 45$ & -1.20 & $-2.54^{\mathrm{d}}$ \\
\hline TIMP3 & & 1035_g_at & $788 \pm 105$ & $704 \pm 120$ & $327 \pm 21$ & -1.12 & $-2.41^{\mathrm{d}}$ \\
\hline TIMP4 & & 819_at & $68 \pm 8$ & $68 \pm 10$ & $68 \pm 7$ & 1.01 & -1.00 \\
\hline$M M P 1$ & Interstitial collagenase & $384 \overline{2} 8$ at & $39 \pm 8$ & $36 \pm 2$ & $36 \pm 4$ & 1.05 & 1.03 \\
\hline$M M P 2$ & Gelatinase A & 39007_at & $68 \pm 8$ & $71 \pm 4$ & $70 \pm 14$ & 1.05 & 1.03 \\
\hline$M M P 11$ & Stromelysin 3 & 38181_at & $69 \pm 9$ & $68 \pm 10$ & $65 \pm 7$ & -1.01 & -1.05 \\
\hline MMP14 & $M T 1-M M P$ & 160020_at & $953 \pm 175$ & $928 \pm 34$ & $861 \pm 129$ & -1.03 & -1.11 \\
\hline$M M P 25$ & MT6-MMP & 35910_f_at & $157 \pm 14$ & $157 \pm 9$ & $149 \pm 18$ & -1.00 & -1.05 \\
\hline$M M P 25$ & MT6-MMP & 35911_r_at & $104 \pm 13$ & $105 \pm 16$ & $98 \pm 18$ & 1.01 & -1.06 \\
\hline
\end{tabular}

${ }^{a}$ Affymetrix identification numbers correspond to independent gene representations on the HG-U95Av2 GeneChip.

${ }^{\mathrm{b}}$ Average hybridisation intensities and standard deviations were derived from four replicate hybridisation intensity values normalised using the dChip PM-only model.

${ }^{\mathrm{c}}$ Treatment conditions were $100 \mu \mathrm{g}$ protein/ml for $24 \mathrm{~h}$.

${ }^{\mathrm{d}}$ Adjusted $p<0.05$ (Bonferroni method) for two-tailed unpaired Student's $t$ test. 

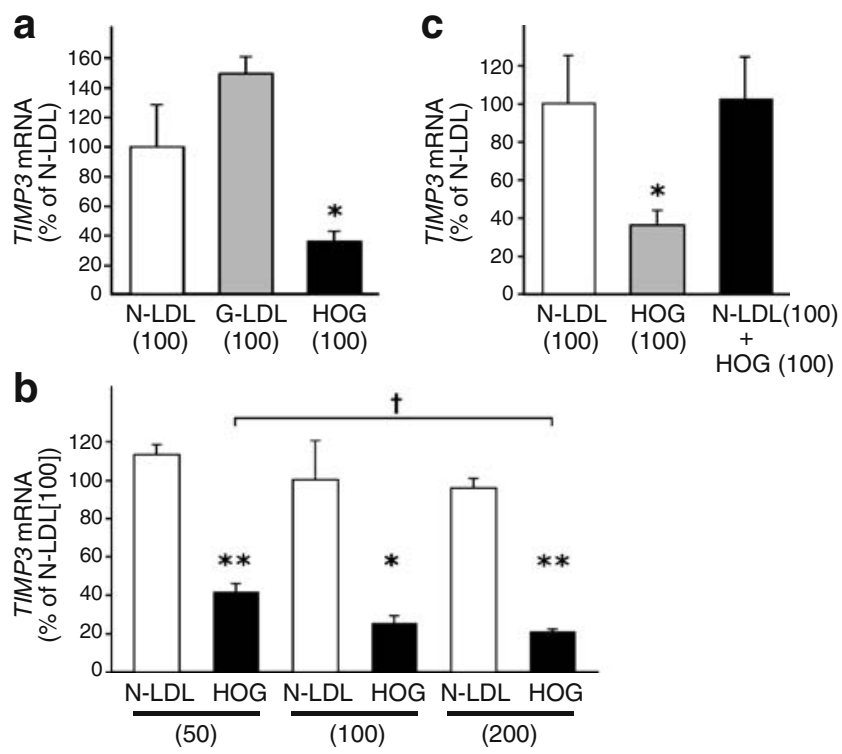

Fig. 1 Quantitative PCR analysis of TIMP3 expression in human retinal pericytes in response to native and modified LDL. The amount of TIMP3 expression by N-LDL $(100 \mu \mathrm{g}$ protein $/ \mathrm{ml})$ treated cells was designated as $100 \%$. Values represent means of triplicate samples and SD for experiments performed at least twice. Significant differences either vs N-LDL treatment or between conditions as indicated: ${ }^{*} p<$ $0.05, * * p<0.01$. a Cells were treated with $\mathrm{N}-, \mathrm{G}-$ or HOG-LDL (HOG) at $100 \mu \mathrm{g}$ protein $/ \mathrm{ml}$ in SFM for $24 \mathrm{~h}$. Note that TIMP3 expression in response to HOG-LDL was significantly reduced (by $68 \%$ ) compared with N-LDL. b Cells were treated with N- or HOGLDL (HOG) at 50, 100 or $200 \mu \mathrm{g}$ protein $/ \mathrm{ml}$ in SFM for $24 \mathrm{~h}(\mathrm{~N}$ LDL100 normalised to $100 \%$ ). TIMP3 expression in response to three concentrations of N-LDL was similar; however, its expression was significantly reduced by HOG-LDL in a concentration-related fashion (significant difference between 50 and $200 \mu \mathrm{g}$ protein/ml HOG-LDL, $\dagger p<0.05$ ). c Cells were treated with N-LDL, HOG-LDL (HOG) or NLDL plus HOG-LDL at $100 \mu \mathrm{g}$ protein/ml each in SFM for $24 \mathrm{~h}$. Note that reduced TIMP3 expression in response to HOG-LDL was completely reversed by co-incubation of N-LDL

Fig. 1a). G-LDL treatment also resulted in a moderate reduction in TIMP3 compared with N-LDL, but the difference was not significant. As outlined above, experiments to measure TIMP3 in conditioned medium revealed that TIMP3 was undetectable before or after treatment with modified LDL, indicating that, as expected, it is not secreted into culture medium.

TIMP1, TIMP2 and MMP1 secretion was not decreased on exposure to HOG-LDL Unlike TIMP3, TIMP-1 and TIMP2 are found almost exclusively in the medium of human retinal cell cultures [19]. Therefore, to confirm the microarray findings that HOG-LDL does not affect the expression of TIMP1 and TIMP2, the secretion of TIMP-1 and TIMP-2 by pericytes in response to modified LDL was measured by ELISA. As shown in Fig. $2 \mathrm{~b}$ and $\mathrm{c}$, secretion of TIMP1 by pericytes treated with G- or HOG-LDL did not differ significantly from secretion by N-LDL-treated cells. Secretion of TIMP2 was modestly inhibited by G- a
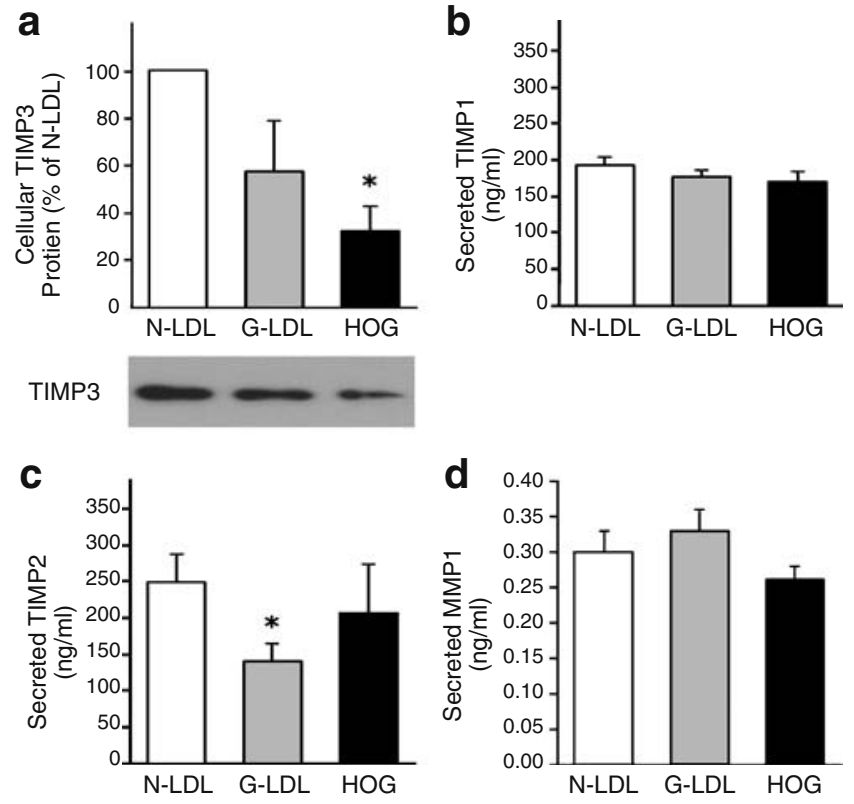

Fig. 2 TIMP1, TIMP2 and TIMP3 and MMP1 protein levels in human retinal pericytes in response to native and modified LDL. Retinal pericytes were treated with $\mathrm{N}-$, G- or HOG-LDL (HOG) at $100 \mu \mathrm{g}$ protein $/ \mathrm{ml}$ in SFM for $24 \mathrm{~h}$. Values represent means of triplicate samples and SD for experiments performed at least twice. Significant differences with regard to the N-LDL treatment: ${ }^{*} p<$ 0.05). a Detection of TIMP3 in cellular/matrix extracts. Upper panel shows averages of normalised densitometric values collected from two immunoblot experiments (N-LDL response defined as $100 \%$ ); lower panel shows the result from one of the TIMP3 immunoblot detection experiments. Note that HOG-LDL significantly reduced TIMP3 level vs N-LDL treatment. b-d ELISA assay of secreted TIMP1, TIMP2 and MMP1. Conditioned medium was collected for analysis. Note that, compared with N-LDL, HOG-LDL did not significantly affect the levels of TIMP1, TIMP2 or MMP1. G-LDL decreased TIMP2 level modestly (by $\sim 40 \%$ )

LDL $(p<0.05)$, but not by HOG-LDL. We did not measure TIMP4 protein because it has been reported to be predominantly produced in the heart [20], and its mRNA expression in pericytes was much lower than that of the genes encoding the other three TIMPs (Table 1).

Previous studies have shown that MMP1 is upregulated in vascular endothelial and smooth muscle cells by oxidised LDL $[21,22]$. Therefore, we examined the effects of HOGLDL on MMP1 secretion by cultured pericytes. The results showed that MMP1 secretion by pericytes was low, and that exposure to HOG- or G-LDL vs N-LDL had no significant effects (Fig. 2d). Again, this is in agreement with the microarray findings, and supports the conclusion that TIMP3 expression is specifically reduced after HOG-LDL treatment.

Collagenase activity in conditioned culture medium was not affected by HOG-LDL Since TIMP3 is released into the ECM but not into conditioned culture medium [19], a reduction in TIMP3 levels caused by HOG-LDL is not expected to affect the collagenase activity in conditioned 
culture medium. Nevertheless, we measured the overall collagenase activity in conditioned medium to exclude the possibility that HOG-LDL might alter the levels or activity of MMPs or other members of the TIMP family. As expected, no difference was observed in collagenase activity in medium conditioned by HOG- vs N-LDLtreated cells (data not shown).

\section{Discussion}

Clinical studies, including the DCCT/Epidemiology of Diabetes Interventions and Complications Study (EDIC) $[23,24]$ and the Hoorn study [25], have revealed an association between dyslipidaemia and diabetic microvascular complications, which is corroborated by the beneficial effects of lipid-lowering therapy on diabetic retinopathy and nephropathy [26]. Evidence also suggests that dyslipidaemia may promote retinal microvascular inflammation and the formation of pericyte ghosts in diabetes, independently of hyperglycaemia [27, 28]. Modification of LDL by glycation and oxidation represents a qualitative dyslipidaemia that is accentuated in both type 1 and type 2 diabetes [9-11]. Although a role of modified LDL in the development of macrovascular disease (atherosclerosis) is well documented, its involvement in diabetic retinopathy is less clear. Lupo et al. [29] reported that oxidised LDL induced phospholipid hydrolysis, inflammation and cytotoxicity in bovine retinal pericytes. Our earlier work showed cytotoxic effects of modified LDL on both human and bovine retinal pericytes and endothelial cells $[13,15]$. To identify the initial changes in human retinal pericytes in response to modified LDL, we performed a microarray study that identified 60 genes whose expression varied $\geq 1.7$-fold in response to HOG-LDL compared with N-LDL [16]. Of these, TIMP3 showed expression that was significantly decreased by HOGcompared with N-LDL, suggesting a possible alteration of the TIMP-MMP balance. Here we conducted a comprehensive evaluation of the effects of modified LDL on the expression of mRNAs of the genes encoding TIMPs and MMPs and the production of TIMP and MMP proteins in retinal pericytes.

Our results showed that HOG-LDL, at a sublethal concentration $(100 \mu \mathrm{g}$ protein $/ \mathrm{ml})$, significantly reduced TIMP3 mRNA expression by $\sim 60 \%$ and protein production by a similar amount in cultured human retinal pericytes, suggesting that the regulation occurs at the transcriptional level. Separate experiments showed that HOG-LDL affected TIMP3 expression in a concentration-dependent manner. The markedly decreased expression of TIMP3 after exposure to HOG-LDL compared with N-LDL was observed consistently in more than 12 separate experiments. Interestingly, the addition of HOG-LDL did not affect expression of the genes encoding the other three members of the TIMP family or any of the detectable MMPs in pericytes.

We also employed lipoprotein-free control conditions, both SFM and RSM (0.5\%). With SFM, TIMP3 expression was rather variable compared with N-LDL. RSM is perhaps a preferable control condition, since the retina is a metabolically active, nutrient-rich environment, and in this, TIMP3 expression was similar in the presence and absence of N-LDL.

We found that a reduction in the TIMP3 protein level in pericytes after HOG-LDL treatment did not lead to an overall change in collagenase activity in the conditioned culture medium. This is consistent with our finding that TIMP3 was not detectable in the conditioned medium in which MMPs were present, and with the previous finding that TIMP3 secreted from pericytes is bound to and forms an insoluble complex with ECM constituents, and is not released into conditioned medium [19]. Thus, TIMP3 may not be able to bind MMPs in this in vitro setting.

The pathophysiologically relevant concentrations of modified LDL in retinae are unknown. Pericytes are traditionally believed not to be in direct contact with the circulation. However, capillary leakage occurs at early stages of diabetic retinopathy, and extravasation and entrapment of LDL in the subendothelial space may allow continuing glycation and oxidation, leading to local accumulation of various concentrations of severely modified particles. Recent histological evidence in the retina of diabetic patients revealed diffuse lipids and cholesteryl ester as well as co-localised ApoB and macrophages in the perivascular space, suggesting LDL extravasation [14]. Qaum et al. [30] demonstrated in rats that, after induction of diabetes, the retinal vasculature is permeable to microspheres as large as $100 \mathrm{~nm}$ in diameter; in comparison, LDL is much smaller, with a diameter of $\sim 20 \mathrm{~nm}$. Furthermore, accumulation of LDL in the vessel wall may be particularly high, as evidenced by the finding of more than a twofold higher level of LDL within the intima of normal human aortas than in plasma [31], suggesting active transport or retention of LDL across the endothelium. Given the complexity of this situation, we chose the LDL concentration range of 50-200 $\mu \mathrm{g}$ protein $/ \mathrm{ml}$ to test the concentrationresponse relations and used $100 \mu \mathrm{g}$ protein $/ \mathrm{ml}$ for additional analysis. The latter dose reflects a very conservative estimate of conditions in vivo, since the concentration range of $\mathrm{ApoB}$ in normal plasma is $700-1200 \mu \mathrm{g} / \mathrm{ml}$.

Our present results are of potential clinical significance. TIMPs inhibit most, if not all, MMPs [1]; a reduction in TIMP3 expression without changes in MMPs may cause an imbalance between TIMPs and MMPs, potentially leading to various consequences, such as matrix remodelling. For example, a reduction in TIMP3 levels may result in adverse matrix remodelling in the cardiomyopathic hamster and the failing human heart [32]. It has been shown that retinal 
pericytes interact closely with endothelial cells, through at least three types of junction, including peg and socket arrangements, adhesion plaques, and cell-cell contacts via adjacent cellular membranes in the basal lamina [33, 34]. These morphological connections and the proximity of pericytes to endothelial cells in retinal as well as many other microvascular beds led to the hypothesis that pericytes regulate endothelial cell function [35], and this has been substantiated by a number of later studies [36-38]. This regulation is of particular importance in the specialised retinal vasculature, where pericyte coverage is greater than in other capillaries [39, 40]. In diabetic retinopathy, pericyte dropout, loss of pericyte-endothelial cell contacts and basal lamina thickening are all observed [41, 42], suggesting an essential role for pericytes in controlling endothelial cell homeostasis. Although our present finding has not been tested in a co-culture or in vivo system, a recent study by Saunders et al. [43] showed that, in three-dimensional collagen matrices, interactions of vascular endothelial cells with bovine retinal pericytes strongly induced TIMP3 expression by pericytes. Further, suppression of pericyte TIMP3 expression using small interfering RNAs led to capillary tube regression in these co-cultures in a MMPdependent manner, and mutagenesis experiments revealed that TIMP3 proteinase inhibitory functions were responsible for tube stabilisation. This evidence strongly suggests that TIMP3 in the pericyte plays a critical role in stabilising endothelial cells and vascular networks. Therefore, a decrease in pericyte expression of TIMP3 in vivo caused by HOG-LDL could elicit endothelial cell instability or proliferation leading to vascular assembly. In addition, a reduced TIMP3 level may augment VEGF-stimulated angiogenesis [44]. Even though pericytes may be lost almost entirely by the late stages of retinopathy, their interaction with LDL very early in the disease process might set the stage for the progression of retinopathy by inducing early changes in the extracellular matrix.

Of the four known TIMPs, TIMP3 is unique in that it inhibits several ADAM (a disintegrin and metalloproteinase) proteins [1]. Recently, a relationship between TIMP3 and TNF has been established. In TIMP3-deficient animal models, abnormal TNF activities were detected that led to enhanced inflammation [45]. The association of TIMP3 with inflammation is of particular interest, given that chronic inflammation has been linked to diabetic retinopathy [46]. Federici et al. [47] reported that TIMP3 deficiency in insulin receptor-haploinsufficient mice promoted diabetes and vascular inflammation via increased TNF- $\alpha$. Therefore, the unique response of TIMP3 to modified LDL seen here suggests an additional mechanism by which modified LDL may promote diabetic retinopathy.

Federici et al. [48] also reported significantly lower TIMP3 expression in carotid atherosclerotic plaques from patients with abnormal glucose tolerance or type 2 diabetes. This suggests that inhibition of TIMP 3 gene expression may also be a feature of macrovascular disease in diabetes. Fabunmi et al. [49], however, found that extracts of carotid atheroma appeared to contain a higher level of TIMP3 than non-atherosclerotic tissue, and suggested that increased TIMP3 might serve as a protective mechanism against plaque rupture. Despite the divergent findings, these studies illustrate the importance of TIMP3 in the development of macrovascular disease, and suggest that the diabetic environment may affect the expression of TIMP3.

The mechanism by which HOG-LDL modulates the expression of TIMP3 is unclear, but the data suggest that oxidation of LDL is crucial for producing the modulation of TIMP3 expression. This is consistent with our earlier findings demonstrating that HOG-LDL is significantly more cytotoxic than G-LDL [13] and demonstrating a distinct pattern of global gene expression induced by HOGLDL, but not by G-LDL, in human retinal pericytes [16]. Our group has reported that uptake of HOG-LDL in rat mesangial cells mainly occurs via scavenger receptors, whereas N- and G-LDL are taken up by the LDL receptors [18]. Whether the differential actions of HOG- and G-LDL can be explained by their separate receptor mechanisms is not known. Our present results showed that the altered TIMP3 expression was completely restored by combined $\mathrm{N}-$ and HOG-LDL treatments, suggesting that TIMP3 expression is likely to be contingent on N-LDL and its interaction with the LDL receptor. More work on the receptor mechanisms mediating pericyte-LDL interactions is needed.

In summary, we demonstrated that HOG-LDL specifically reduces the expression of TIMP3 and production of TIMP3 protein in human retinal pericytes. This response was not observed for any other TIMPs and MMPs detectable in pericytes. Since TIMP3 is a potent inhibitor of MMPs and, unlike other TIMPs, also exhibits other unique properties, a reduction in TIMP3 production by pericytes as a consequence of LDL modification may contribute to the development of diabetic retinopathy.

Acknowledgement This work was supported by an American Diabetes Association research grant to T. J. Lyons, a VA Merit Review grant to Y. Huang and NIH grants HL61873 and CA095841 to W. S. Argraves. Analysis of DNA microarray data was conducted using resources of the MUSC Proteogenomics Facility (http://www. proteogenomics.musc.edu/), which is supported by grants from the National Cancer Institute (CA 095841) and the National Heart Lung and Blood Institute (P20RR016434). It was also supported by NIH Grant M01 RR-14467 to the General Clinical Research Center at University of Oklahoma Health Sciences Center.

Duality of interest The authors confirm that there is no duality of interest associated with this manuscript. 


\section{References}

1. Visse R, Nagase H (2003) Matrix metalloproteinases and tissue inhibitors of metalloproteinases: structure, function, and biochemistry. Circ Res 92:827-839

2. An E, Lu X, Flippin J et al (2006) Secreted proteome profiling in human RPE cell cultures derived from donors with age related macular degeneration and age matched healthy donors. J Proteome Res 5:2599-25610

3. Plantner JJ, Jiang C, Smine A (1998) Increase in interphotoreceptor matrix gelatinase A (MMP-2) associated with age-related macular degeneration. Exp Eye Res 67:637-645

4. Weber BH, Vogt G, Pruett RC, Stohr H, Felbor U (1994) Mutations in the tissue inhibitor of metalloproteinases-3 (TIMP3) in patients with Sorsby's fundus dystrophy. Nat Genet 8:352-356

5. Grant MB, Caballero S, Tarnuzzer RW et al (1998) Matrix metalloproteinase expression in human retinal microvascular cells. Diabetes 47:1311-1317

6. Das A, McGuire PG, Eriqat C et al (1999) Human diabetic neovascular membranes contain high levels of urokinase and metalloproteinase enzymes. Invest Ophthalmol Vis Sci 40:809-813

7. Giebel SJ, Menicucci G, McGuire PG, Das A (2005) Matrix metalloproteinases in early diabetic retinopathy and their role in alteration of the blood-retinal barrier. Lab Invest 85:597-607

8. Descamps FJ, Martens E, Kangave D et al (2006) The activated form of gelatinase B/matrix metalloproteinase-9 is associated with diabetic vitreous hemorrhage. Exp Eye Res 83:401-407

9. Lyons TJ, Baynes JW, Patrick JS, Colwell JA, Lopes-Virella MF (1986) Glycosylation of low density lipoprotein in patients with type 1 (insulin-dependent) diabetes: correlations with other parameters of glycaemic control. Diabetologia 29:685-689

10. Tsai EC, Hirsch IB, Brunzell JD, Chait A (1994) Reduced plasma peroxyl radical trapping capacity and increased susceptibility of LDL to oxidation in poorly controlled IDDM. Diabetes 43:1010-1014

11. Paniagua JA, Lopez-Miranda J, Perez-Martinez P et al (2005) Oxidized-LDL levels are changed during short-term serum glucose variations and lowered with statin treatment in early type 2 diabetes: a study of endothelial function and microalbuminuria. Diabet Med 22:1647-1656

12. Chisolm GM, Steinberg D (2000) The oxidative modification hypothesis of atherogenesis: an overview. Free Radic Biol Med 28:1815-1826

13. Song W, Barth JL, Lu K et al (2005) Effects of modified lowdensity lipoproteins on human retinal pericyte survival. Ann N Y Acad Sci 1043:1-6

14. Cusick M, Chew EY, Chan CC, Kruth HS, Murphy RP, Ferris FL III (2003) Histopathology and regression of retinal hard exudates in diabetic retinopathy after reduction of elevated serum lipid levels. Ophthalmology 110:2126-2133

15. Lyons TJ, Li W, Wells-Knecht MC, Jokl R (1994) Toxicity of mildly modified low-density lipoproteins to cultured retinal capillary endothelial cells and pericytes. Diabetes 43:1090-1095

16. Song W, Barth JL, Yu Y et al (2005) Effects of oxidized and glycated LDL on gene expression in human retinal capillary pericytes. Invest Ophthalmol Vis Sci 46:2974-2982

17. Li C, Wong WH (2001) Model-based analysis of oligonucleotide arrays: expression index computation and outlier detection. Proc Natl Acad Sci USA 98:31-36

18. Jenkins AJ, Velarde V, Klein RL et al (2000) Native and modified LDL activate extracellular signal-regulated kinases in mesangial cells. Diabetes 49:2160-2169

19. Vranka JA, Johnson E, Zhu X et al (1997) Discrete expression and distribution pattern of TIMP-3 in the human retina and choroid. Curr Eye Res 16:102-110
20. Greene J, Wang M, Liu YE, Raymond LA, Rosen C, Shi YE (1996) Molecular cloning and characterization of human tissue inhibitor of metalloproteinase 4. J Biol Chem 271:30375-30380

21. Huang Y, Mironava M, Lopes-Virella MF (1999) Oxidized LDL stimulates matrix metalloproteinase-1 expression in human vascular endothelial cells. Arterioscler Thromb Vasc Biol 19:2640-2647

22. Game BA, Maldonado A, He L, Huang Y (2005) Pioglitazone inhibits MMP-1 expression in vascular smooth muscle cells through a mitogen-activated protein kinase-independent mechanism. Atherosclerosis 2005 178:249-256

23. Lyons TJ, Jenkins AJ, Zheng D et al (2004) Diabetic retinopathy and serum lipoprotein subclasses in the DCCT/EDIC cohort. Invest Ophthalmol Vis Sci 45:910-918

24. Jenkins AJ, Lyons TJ, Zheng D et al (2003) Lipoproteins in the DCCT/EDIC cohort: associations with diabetic nephropathy. Kidney Int 64:817-828

25. van Leiden HA, Dekker JM, Moll AC et al (2002) Blood pressure, lipids, and obesity are associated with retinopathy: the Hoorn study. Diabetes Care 25:1320-1325

26. Misra A, Kumar S, Kishore Vikram N, Kumar A (2003) The role of lipids in the development of diabetic microvascular complications: implications for therapy. Am J Cardiovasc Drugs 3:325-338

27. Chen W, Jump DB, Grant MB, Esselman WJ, Busik JV (2003) Dyslipidemia, but not hyperglycemia, induces inflammatory adhesion molecules in human retinal vascular endothelial cells. Invest Ophthalmol Vis Sci 44:5016-5022

28. Barile GR, Pachydaki SI, Tari SR et al (2005) The RAGE axis in early diabetic retinopathy. Invest Ophthalmol Vis Sci 46:2916-2924

29. Lupo G, Anfuso CD, Ragusa N, Strosznajder RP, Walski M, Alberghina M (2001) $t$-Butyl hydroperoxide and oxidized low density lipoprotein enhance phospholipid hydrolysis in lipopolysaccharide-stimulated retinal pericytes. Biochim Biophys Acta 1531:143-155

30. Qaum T, Xu Q, Joussen AM et al (2001) VEGF-initiated bloodretinal barrier breakdown in early diabetes. Invest Ophthalmol Vis Sci 42:2408-2413

31. Smith EB, Staples EM (1982) Plasma protein concentrations in interstitial fluid from human aortas. Proc R Soc Lond B Biol Sci 217:59-75

32. Fedak PW, Altamentova SM, Weisel RD et al (2002) Matrix remodeling in experimental and human heart failure: a possible regulatory role for TIMP-3. Am J Physiol Heart Circ Physiol 284: H626-H634

33. Leeson TS (1979) Rat retinal blood vessels. Can J Ophthalmol $14: 21-28$

34. Carlson EC (1989) Fenestrated subendothelial basement membranes in human retinal capillaries. Invest Ophthalmol Vis Sci 30:1923-1932

35. Crocker DL, Murad TM, Geer JC (1970) Role of the pericyte in wound healing. An ultrastructural study. Exp Mol Pathol 13:90-94

36. Orlidge A, D'Amore PA (1987) Inhibition of capillary endothelial cell growth by pericytes and smooth muscle cells. J Cell Biol 105:1455-1462

37. Sato Y, Rifkin DB (1989) Inhibition of endothelial cell movement by pericytes and smooth muscle cells: activation of a latent transforming growth factor-beta-like molecule by plasmin during co-culture. J Cell Biol 109:309-315

38. Watanabe S, Morisaki N, Tezuka M et al (1997) Cultured retinal pericytes stimulate in vitro angiogenesis of endothelial cells through secretion of a fibroblast growth factor-like molecule. Atherosclerosis 130:101-107

39. Shepro D, Morel NM (1993) Pericyte physiology. FASEB J 7:1031-1038

40. Hammes HP (2005) Pericytes and the pathogenesis of diabetic retinopathy. Horm Metab Res 37(Suppl 1):39-43 
41. Kuwabara T, Cogan DG (1963) Retinal vascular patterns. VI. Mural cells of the retinal capillaries. Arch Ophthalmol 69:492-502

42. Robison WG Jr, Nagata M, Tillis TN, Laver N, Kinoshita JH (1989) Aldose reductase and pericyte-endothelial cell contacts in retina and optic nerve. Invest Ophthalmol Vis Sci 30:2293-2299

43. Saunders WB, Bohnsack BL, Faske JB (2006) Coregulation of vascular tube stabilization by endothelial cell TIMP-2 and pericyte TIMP-3. J Cell Biol 175:179-191

44. Qi JH, Ebrahem Q, Moore N et al (2003) A novel function for tissue inhibitor of metalloproteinases-3 (TIMP3): inhibition of angiogenesis by blockage of VEGF binding to VEGF receptor-2. Nat Med 9:407-415

45. Mohammed FF, Smookler DS, Taylor SE et al (2004) Abnormal TNF activity in Timp3-/- mice leads to chronic hepatic inflammation and failure of liver regeneration. Nat Genet 36:969-977
46. Joussen AM, Poulaki V, Le ML et al (2004) A central role for inflammation in the pathogenesis of diabetic retinopathy. FASEB J 18:1450-1452

47. Federici M, Hribal ML, Menghini R et al (2005) Timp3 deficiency in insulin receptor-haploinsufficient mice promotes diabetes and vascular inflammation via increased TNF-alpha. J Clin Invest 115:3494-3505

48. Federici M, Menghini R, Perego L et al (2006) Reduced TIMP3 levels in atherosclerotic plaques from patients affected by altered glucose tolerance. ADA Annual Scientific Sessions Abstracts, 657-P (Abstr)

49. Fabunmi RP, Sukhova GK, Sugiyama S, Libby P (1998) Expression of tissue inhibitor of metalloproteinases-3 in human atheroma and regulation in lesion-associated cells: a potential protective mechanism in plaque stability. Circ Res 83:270-278 